\title{
Evolution of Oscillating Scalar Fields as Dark Energy
}

\author{
Sourish Dutta and Robert J. Scherrer \\ Department of Physics and Astronomy, Vanderbilt University, Nashville, TN 37235
}

\begin{abstract}
Oscillating scalar fields, with an oscillation frequency much greater than the expansion rate, have been proposed as models for dark energy. We examine these models, with particular emphasis on the evolution of the ratio of the oscillation frequency to the expansion rate. We show that this ratio always increases with time if the dark energy density declines less rapidly than the background energy density. This allows us to classify oscillating dark energy models in terms of the epoch at which the oscillation frequency exceeds the expansion rate, which is effectively the time at which rapid oscillations begin. There are three basic types of behavior: early oscillation models, in which oscillations begin during the matter-dominated era, late oscillation models, in which oscillations begin after scalar-field domination, and non-oscillating models. We examine a representative set of models (those with power-law potentials) and determine the parameter range giving acceptable agreement with the supernova observations. We show that a subset of all three classes of models can be consistent with the observational data.
\end{abstract}

\section{INTRODUCTION}

It appears that approximately $70 \%$ of the energy density in the universe is in the form of an exotic, negativepressure component, dubbed dark energy [1, 2, 3, 4]. (See Ref. [5] for a recent review). The most likely possibility for this dark energy is a cosmological constant. Another possibility, dubbed quintessence, is a model in which the dark energy arises from a scalar field $[6,67,8,9,010]$.

Although it is usually assumed that the evolution of the scalar field is monotonic, a more exotic possibility is that the scalar field oscillates. Such oscillating scalar fields were first investigated by Turner [11], and later reexamined in the context of models for dark energy [9, 12, 13, 14, 15]. (See also Ref. [16] for a phantom version of these models, and Ref. [17] for applications to inflation). Here we examine a key aspect of such models: the interplay between the oscillation frequency and the Hubble expansion rate. The discussions in Refs. [11, 12, 13, 14, 15] assumed that the oscillation frequency is large compared to the expansion rate. However, if the ratio between these two quantities crosses unity, it is possible to generate interesting new behaviors for the dark energy. We explore this possibility here. (Note that the models considered here, in which the scalar field oscillates, are distinct from models in which the equation of state oscillates [18, 19, 20, 21, 22]. In the latter case, one is generally interested in oscillation frequencies which are always on the order of the Hubble parameter. In the models considered here, oscillations with frequencies on the order of the Hubble parameter are a transient, albeit important, phenomenon).

In the next section, we review the evolution of oscillating scalar fields in an expanding background, and we derive some interesting general results on the evolution of the ratio of scalar field oscillation frequency to the expansion rate. In Sec. III, we compare numerical simulations of a representative set of oscillating models (due to power-law potentials) with the observations. Our conclusions are summarized in Sec. IV. Our main new result is the existence of a class of oscillating models in which the scalar field is initially slowly rolling, but oscillates at late times; we show that such models can be consistent with the observations, but this result is very sensitive to the phase of the scalar field oscillation at the present.

\section{EVOLUTION OF OSCILLATING SCALAR FIELDS}

\section{A. Previous work}

Consider a minimally-coupled scalar field, $\phi$, in a potential $V(\phi)$. The equation of motion for this field in an expanding background is

$$
\ddot{\phi}+3 H \dot{\phi}+\frac{d V}{d \phi}=0
$$

where the Hubble parameter $H$ is given by

$$
H=\left(\frac{\dot{a}}{a}\right)=\sqrt{\rho_{T} / 3}
$$

Here $a$ is the scale factor, $\rho_{T}$ is the total density, and we work in units for which $8 \pi G=1$. The pressure and density of $\phi$ are given by

$$
p_{\phi}=\frac{\dot{\phi}^{2}}{2}-V(\phi)
$$

and

$$
\rho_{\phi}=\frac{\dot{\phi}^{2}}{2}+V(\phi),
$$

respectively, and the equation of state parameter, $w_{\phi}$, is defined to be

$$
w_{\phi}=p_{\phi} / \rho_{\phi} .
$$

The density of the scalar field then evolves with the scale factor as

$$
\rho_{\phi} \propto a^{-3\left(1+w_{\phi}\right)} .
$$


Turner [11] was the first to consider the behavior of such a field in the limit where it oscillates on a timescale much shorter than the Hubble time. He examined powerlaw potentials of the form

$$
V(\phi)=k \phi^{n}
$$

and he showed that the value of $w_{\phi}$, averaged over the oscillation period, is

$$
w_{\phi}=(n-2) /(n+2) .
$$

Thus, the cases $n=2$ and $n=4$ behave like nonrelativistic matter and radiation, respectively.

Liddle and Scherrer [9] investigated oscillating scalar field models with power-law potentials in the context of dark energy, noting an interesting set of behaviors: for a universe dominated by a background fluid with equation of state parameter $w_{B}$, the oscillating solution is an attractor of the equations of motion only for the case

$$
n<\frac{2\left(3+w_{B}\right)}{1-w_{B}},
$$

while potentials with $n>2\left(3+w_{B}\right) /\left(1-w_{B}\right)$ produce a scalar field trajectory that decreases monotonically to zero without oscillating. Liddle and Scherrer provided no physical explanation for this curious behavior; we give such an explanation below.

Sahni and Wang [12] and Hsu [13] examined the possibility that an oscillating field could give rise to dark energy. From equation (8), it is clear that $w<0$ requires $n<2$, and the production of a dark energy equation of state can only be achieved if we take $V=k|\phi|^{n}$, with $n \ll 1$. Although potentials with very small $n$ might appear rather unnatural, such a potential can arise from a Lagrangian with a $\phi^{2}$ potential and a non-standard kinetic term [13].

Masso et al. [14] performed a general investigation of oscillating scalar fields in arbitrary potentials (as opposed to power laws). Using the action-angle formalism, they derived several very important results, which we will use here. Consider a scalar field $\phi$ oscillating in an arbitrary potential $V(\phi)$. The minimum and maximum values of $\phi$ are $\phi_{1}$ and $\phi_{2}$ respectively. Then the energy density for this field must be equal to $\rho_{\phi}=V\left(\phi_{1}\right)=V\left(\phi_{2}\right)$. (Note that $\rho_{\phi}$ changes with time, but it is assumed to evolve on a timescale longer than the inverse oscillation frequency). Masso et al. showed that all of the quantities of interest can be expressed in terms of the action variable $J$, defined by

$$
J=2 \int_{\phi_{1}}^{\phi_{2}} \sqrt{2\left(\rho_{\phi}-V(\phi)\right)} .
$$

Then the oscillation frequency $\nu$ is

$$
\nu=\frac{d \rho_{\phi}}{d J}
$$

while the equation of state parameter is

$$
\begin{aligned}
1+w_{\phi} & =\frac{J}{\rho_{\phi}} \frac{1}{d J / d \rho_{\phi}}, \\
& =\frac{2}{\rho_{\phi}} \frac{\int_{\phi_{1}}^{\phi_{2}}\left[\rho_{\phi}-V(\phi)\right]^{1 / 2} d \phi}{\int_{\phi_{1}}^{\phi_{2}}\left[\rho_{\phi}-V(\phi)\right]^{-1 / 2} d \phi} .
\end{aligned}
$$

(Note that this last result was also derived by Turner 11]). These results allow for the investigation of somewhat more natural (non-power-law) potentials that might serve as dark energy, and several of these are discussed in Ref. [14].

Finally, $\mathrm{Gu}$ [15] revisited the power-law scenario, and calculated the conditions necessary for the oscillation frequency to exceed the expansion rate (our equation 16 below). He used this condition to place constraints on such power-law models.

\section{B. Evolution of oscillation frequency}

Implicit in all of these discussions is the assumption that the oscillation frequency is much greater than the Hubble expansion rate. We now consider this condition in more detail. The relevant quantity is the ratio of oscillation frequency to Hubble parameter, $\nu / H$. If $\nu / H \gg 1$, then the parameters of interest can be averaged over the oscillation period, as in Refs. [9, 11, 12, 13, 14, 15]. On the other hand, when $\nu / H \ll 1$, the field effectively ceases to oscillate, and an alternative treatment is required.

Consider the evolution of $\nu / H$. From equations (10) and (11) we can write

$$
\nu=\left[\int_{\phi_{1}}^{\phi_{2}} \sqrt{\frac{2}{\rho_{\phi}-V(\phi)}} d \phi\right]^{-1} .
$$

This equation, along with the expression for $H$ given by equation (2), gives us

$$
\nu / H=\left[\int_{\phi_{1}}^{\phi_{2}} \sqrt{\frac{2 \rho_{T}}{3\left(\rho_{\phi}-V(\phi)\right)}} d \phi\right]^{-1} .
$$

For the case of the power-law potential, $V(\phi)=k \phi^{n}$, the expression for $\nu$ can be integrated exactly [14], giving (see also [15])

$$
\nu / H=\frac{\sqrt{3} n \Gamma(1 / 2+1 / n)}{2 \sqrt{2 \pi} \Gamma(1 / n)} \sqrt{\frac{\rho_{\phi}}{\rho_{T}}} \frac{1}{\phi_{\max }},
$$

where $\phi_{\max }=\phi_{1}=\phi_{2}$, since the potential is symmetric. For other symmetric potentials, we can use equation (15) to derive an order of magnitude estimate:

$$
\nu / H \sim \sqrt{\frac{\rho_{\phi}}{\rho_{T}}} \frac{1}{\phi_{\max }} .
$$


This result is clearly consistent with the exact result for power-law potentials given by equation (16).

Consider first the case where the scalar field itself dominates the expansion, so that $\rho_{T} \approx \rho_{\phi}$. In this case, we have $\nu / H \sim 1 / \phi_{\max }$. Since $\rho_{\phi}$ always decreases with time, $\phi_{\max }$ is also a decreasing function of time, so that $\nu / H$ always increases with time.

The other possibility is that the universe is dominated by a background fluid (e.g., matter or radiation) with density $\rho_{B}$. In this case, we have $\nu / H \sim$ $\sqrt{\rho_{\phi} / \rho_{B}}\left(1 / \phi_{\max }\right)$. We can again assume that $\phi_{\max }$ decreases with time. In order for $\phi$ to serve as a plausible dark energy candidate, its density must decrease more slowly with the expansion than the density of matter or radiation. Hence, we can conclude that $\rho_{\phi} / \rho_{B}$ increases with time, and once again we have that $\nu / H$ increases with time.

Thus, for any model in which $\phi$ serves as the dark energy today, $\nu / H$ is an increasing function of time, regardless of whether the universe is background-dominated or $\phi$-dominated.

Before examining the implications of this result, we reconsider the behavior discussed in Ref. [9] and described by equation (9). Equations (8) and (9), taken together, imply that scalar field oscillations are an attractor in a universe dominated by a background fluid only for the case where

$$
w_{\phi}<1 / 2+w_{B} / 2 .
$$

Further, for a power-law potential given by equation (7), in the background-dominated case, equation (17) can be expressed as

$$
\nu / H \sim \rho_{B}^{-1 / 2} \rho_{\phi}^{1 / 2-1 / n} .
$$

Applying equation (8), this becomes

$$
\nu / H \sim \rho_{B}^{-1 / 2} \rho_{\phi}^{w_{\phi} /\left(1+w_{\phi}\right)},
$$

which can be rewritten in terms of the scale factor (using equation (6) for $\rho_{\phi}$ and the corresponding equation for $\left.\rho_{B}\right)$ as

$$
\nu / H \sim a^{3\left(1 / 2+w_{B} / 2-w_{\phi}\right)} .
$$

A comparison of equations (21) and (18) provides a physical explanation for the bound noted by Liddle and Scherrer [9]. When this bound (equation 9) is not satisfied, equation (21) indicates that $\nu / H$ is a decreasing function of time. Thus, at late times, the oscillating solution cannot be an attractor. This is consistent with the behavior noted in Ref. 9]; when equation (9) is not satisfied, the scalar field decays asymptotically to the minimum of the potential without oscillating. These models do not contradict our claim that in any oscillating scalar field model for dark energy, $\nu / H$ always increases with time. The reason is that models that violate the bound in equation (91) have an energy density that decays more rapidly than $\rho_{B}$; they are not plausible models for dark energy.
Now consider the possible oscillating scalar field models for dark energy. The fact that $\nu / H$ increases with time simplifies the classification of such models. If we assume that the universe evolves from a matter-dominated state to a scalar-field dominated state, there are basically three possibilities, which depend on the redshift at which $\nu$ becomes greater than $H$ :

I. The oscillation frequency becomes greater than the expansion rate during the matter-dominated era. Since $\nu / H$ increases with time, we always have $\nu / H>1$ during the quintessence-dominated era, and we can use timeaveraged quantities to describe the equation of state of the quintessence. We will call this the early-oscillation case. This is basically the model described in Refs. 11, 12, 13, 14, 15], so these models have been exhaustively explored.

II. The oscillation frequency is less than $H$ at the present day. Again, since $\nu / H$ always increases with time, this implies that $\nu$ is never larger than $H$, so these models never reach the oscillatory stage. In the language of Ref. [23], these are thawing models in which the field has not yet reached the minimum of the potential. Such models are characterized by a value of $w_{\phi}$ very close to -1 initially, which increases at late times. We will call this the "no oscillation" case. Again, such models have been exhaustively explored (see, e.g., Ref. [24] and references therein).

III. The case intermediate between I and II occurs when $\nu$ becomes greater than $H$ at late times, after the quintessence density exceeds the matter density. In this case, the scalar field displays two distinct behaviors during the quintessence-dominated era. At first, when the oscillation frequency is less than $H$, the scalar field rolls down the potential and $w_{\phi}$ increases. Then, when the oscillation frequency becomes greater than $H$, the field oscillates rapidly, and $w_{\phi}$ takes its time-averaged value as in Refs. 11, 12, 13, 14, 15]. This third possibility has not been previously studied; we will refer to it as the "late oscillation" case.

\section{COMPARISON WITH OBSERVATIONS}

Although we believe that our classification scheme in the previous section should describe most possible models for quintessence from oscillating scalar fields, it is obviously impossible to compare all such models with the observational data. We will therefore limit ourselves to a representative set of models: the power-law oscillating scalar field models discussed in Refs. [12, 13, 14, 15]. As we note below, we believe that the general features of the results we derive for these models will be more generally applicable to other oscillating scalar field models.

Following Ref. [13], we choose the potential to have the form:

$$
V(\phi)=k|\phi|^{n},
$$

where $k$ and $n$ are constants. Then the evolution of the 
field depends on three parameters: $k, n$, and the initial value of $\phi$, which we denote $\phi_{i}$. However, the parameter $k$ can be eliminated from the equation of motion (equation (1) by a suitable rescaling of $\phi$ and the time $t$. This rescaling means that we are no longer using Planck units, but our units of time are irrelevant, since we always define the "present day" in terms of the value of $\Omega_{\phi}$, rather then $t$. (Another way to see this is to note that all of our results depend only on the evolution of $w_{\phi}$ as a function of $\Omega_{\phi}$, and $w_{\phi}\left(\Omega_{\phi}\right)$ depends only on $V^{\prime} / V$, which is independent of $k[10])$. Thus, the evolution can be described entirely in terms of $n$ and $\phi_{i}$.

For a given choice of values of the two free parameters, $\phi_{i}$, and $n$, we numerically evolve the system from a starting point in the matter dominated regime to the point when $\Omega_{m}=0.3$, which we label as the present time $z=0$.

The relation between the evolution of $\phi$ and the two input parameters is illustrated in Fig. 1. In our numerical simulations, we define oscillations to commence when the scalar field crosses $\phi=0$. This corresponds to $w_{\phi}$ increasing from -1 to +1 and marks the point at which $d w_{\phi} / d z$ first changes sign. (Note that the oscillation frequency for $w_{\phi}$ is twice as large as the oscillation frequency for $\phi)$. For early oscillations, this zero-crossing takes place during the matter-dominated era, while for late oscillations it takes place during the quintessence-dominated era. The no oscillation case corresponds to the parameter region in which zero crossing never occurs up to the present.

For a given $n$, the parameter $\phi_{i}$ determines when the oscillations will begin. From equation (16) (or [17), it is clear that small $\phi_{i}$ corresponds to a larger value of $\nu / H$ for a given ratio of $\rho_{\phi}$ to $\rho_{T}$. Thus, oscillations commence earlier if $\phi_{i}$ is small and later if $\phi_{i}$ is large. For fixed $n$, sufficiently small $\phi_{i}$ will produce the early oscillation case, while sufficiently large $\phi_{i}$ gives no oscillations, with late oscillations arising from intermediate values of $\phi_{i}$. This is apparent in Fig. 1. Fixing $n$, we see that increasing $\phi_{i}$ moves the evolution from early oscillation, through late oscillation, and into no oscillation. Since the late oscillation case has not been previously explored, we present a graph, in Fig. 2, of the evolution of $w(z)$ for some of these models. As claimed, $w_{\phi}$ initially evolves slowly upward from $w_{\phi}=-1$, but then oscillates rapidly at late times; this transition takes place after the quintessence field becomes dominant.

Next, we compare the theoretical luminosity-vsdistance results to the recent Type Ia Supernovae standard candle data (ESSENCE+SNLS+HST from [4]). The theoretical distance modulus in our model is calculated in the standard way:

$$
\mu(z)=5 \log _{10} D_{L}(z)+42.38-5 \log _{10} h,
$$

where $h$ is the Hubble parameter in units of $100 \mathrm{~km} \mathrm{sec}^{-1}$ $\mathrm{Mpc}^{-1}$, and $D_{L}(z)$ is the dimensionless luminosity dis-

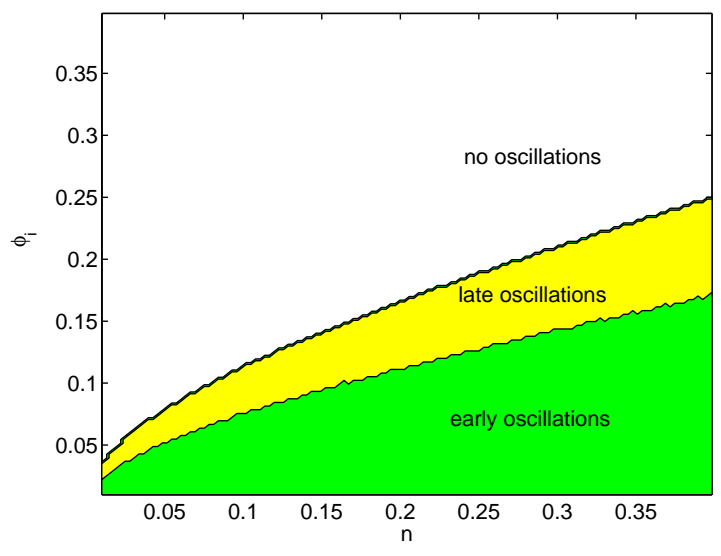

FIG. 1: For the potential $V(\phi)=k|\phi|^{n}$, in the parameter space determined by $n$ and the initial magnitude of the scalar field, $\phi_{i}$, this plot gives the regions where the field $\phi$ oscillates prior to dominating the expansion (early oscillations), after dominating the expansion (late oscillations), or does not oscillate at all prior to the present (no oscillations).

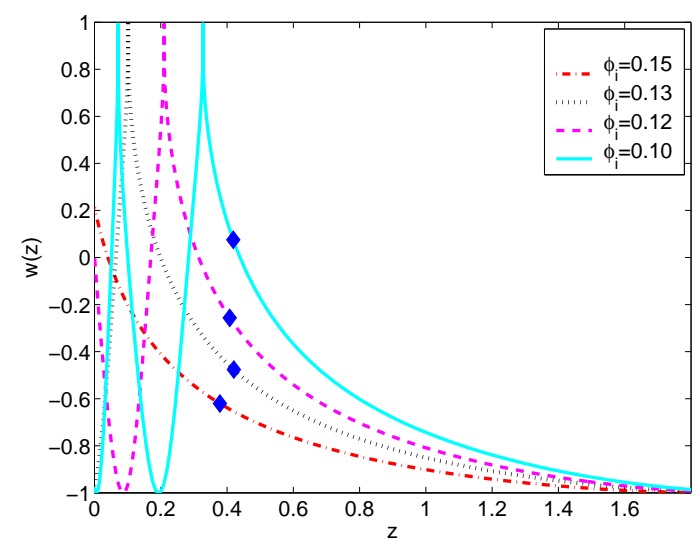

FIG. 2: Evolution of the quintessence equation of state parameter, $w_{\phi}$, as a function of redshift $z$, in the late oscillation regime. Curves correspond to the indicated values of the initial value of the field, $\phi_{i}$, in the power-law potential $V=k|\phi|^{n}$ with $n=0.16$. Diamonds mark the points at which the density of dark energy equals the density of matter. The curve for $\phi_{i}=0.15$ corresponds to the no oscillation regime and has been added for comparison.

tance, given by

$$
D_{L}(z)=(1+z) \int_{0}^{z} \frac{H_{0}}{H\left(z^{\prime}\right)} d z^{\prime},
$$

where $H\left(z^{\prime}\right)$, the Hubble parameter at redshift $z^{\prime}$, also depends on $\Omega_{m 0}$ and the parameters of our quintessence model. We construct a $\chi^{2}$ likelihood plot for $\phi_{i}$ and $n$ ranging from 0.01 to 0.40 (no further structure emerges from extending the parameter space). We take $\Omega_{m 0}=0.3$ and marginalize over the nuisance parameter $h$. Fig. 3 shows this plot.

Some of the allowed regions can be understood in terms of our previous discussion of the behavior of the field. In 


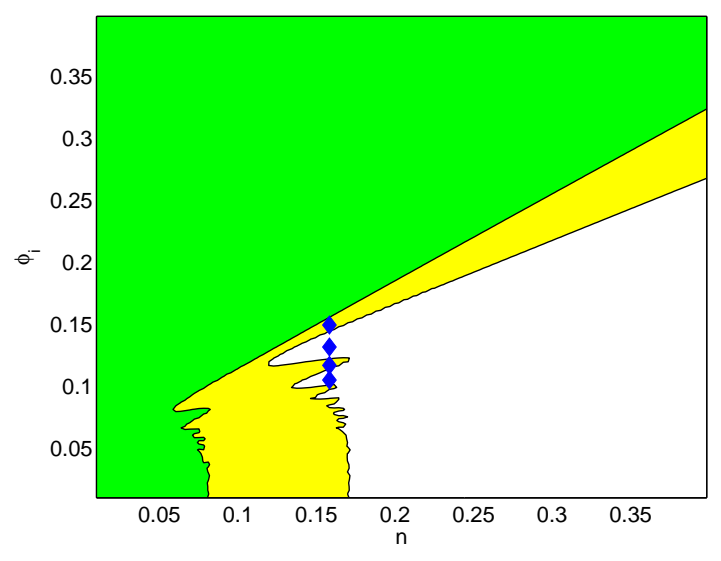

FIG. 3: Likelihood plot for the parameters $\phi_{i}$ and $n$. The $95 \%$ confidence region is green (dark shaded) and the $68 \%$ confidence region is green+yellow (dark shaded + light shaded). The diamonds indicate the models displayed in Figs 2, 5, 6, and 7

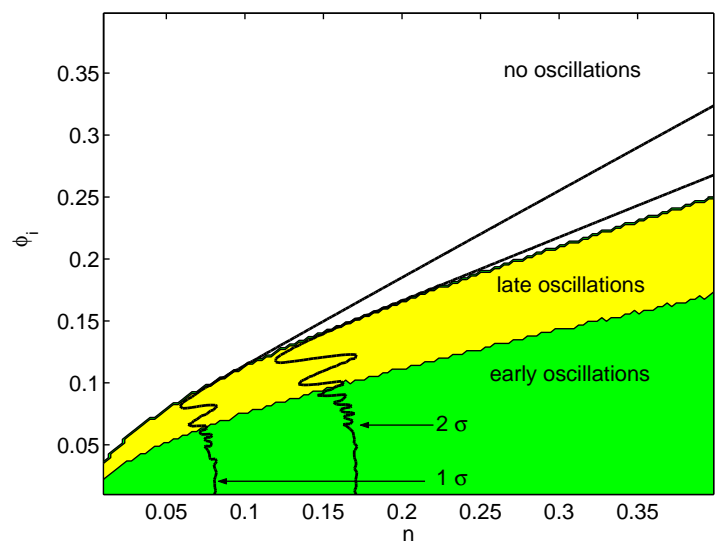

FIG. 4: The likelihood contours of Fig. 3 superimposed over the demarcation of the type of oscillatory behavior from Fig. 1.

the limit of large $\phi_{i}$, the field never oscillates, and it can be effectively treated as a cosmological constant, which is known to be a good fit to the observational data. For sufficiently small $\phi_{i}$, we are in the "early oscillation" regime. In this case, $w_{\phi}$ takes on a constant value determined by equation (8) and independent of $\phi_{i}$. It is clear from equation (8) that a value of $w_{\phi}$ near -1 requires a value of $n$ close to zero. Thus, we see in Fig. 3 that for small $\phi_{i}$, the allowed region is confined to small values of $n$ (which give $w_{\phi}$ near -1 ), and the likelihood contours become vertical lines (indicating that the late-time value of $w_{\phi}$ is independent of $\phi_{i}$ in this limit).

To explore the allowed parameter space further, we have superimposed the field behaviors outlined in Fig. 1 onto the likelihood contours displayed in Fig. 3. The results are displayed in Fig. 4. As noted earlier, almost the entire "no oscillation" parameter space is allowed, while the "early oscillation" parameter space is confined to the region for which $w_{\phi}$ is close to -1 . However, we also see that there is an allowed region in the "late oscillation" regime; this is the subset of oscillating models that has not been previously explored. In these models, the field is initially slowly rolling down the potential, with increasing $w_{\phi}$; it then transitions into rapid oscillations at late times. An interesting feature in this regime is the non-smooth structure of the confidence contours. This is a real effect.

To illustrate the reason for the non-smooth confidence contours, we have sampled four representative models in this regime, denoted by the four diamonds in Fig. 3, which correspond to $n=0.16$ and $\phi_{i}=0.10,0.12,0.13$, and 0.15 . (These are the four models previously displayed in Fig. 2). This illustrates the complex structure of the allowed region as $\phi_{i}=0.10$ and 0.13 are ruled out at $95 \%$ confidence, while $\phi_{i}=0.12$ and 0.15 are not. Next, we display the distance modulus for these four models, along with the supernova data, in Fig. 5] Although the individual models cannot be distinguished here, it is clear that all of these models lie near the edge of the allowed region because they tend to produce a distance modulus that is lower than the observations. In Fig. 6, we show a blow-up of Fig. [5. The smallest values of the distance modulus are produced for $\phi_{i}=0.10$ and 0.13 ; these are the two models that are ruled out at $95 \%$ confidence. The important point here is that the distance modulus is not a monotonic function of $\phi_{i}$. The reason for this is illustrated in Fig. 7, where we give the redshift dependence of the density of the scalar field (which determines $H(z)$, and therefore, $\mu(z))$. Comparing this figure with Fig. 2. we see that $H(z)$ depends crucially on the oscillation phase at the present day. The two models for which the field is at a maximum of the potential at present (thus giving $w_{\phi}=-1$ at present) are $\phi_{i}=0.10$ and $\phi_{i}=0.13$. These produce a larger density at moderate redshift and therefore a smaller integrated value of $1 / H(z)$. Thus, the complex behavior of our contours in Figs. 3 and 4 is a phase effect: the phase of $\phi$ at present is an oscillating function of $\phi_{i}$, and this phase determines whether or not a given model is excluded. For early oscillation models the phase becomes irrelevant, since these models oscillate many times before the present, and the equation of state parameter takes on its period-averaged value.

\section{CONCLUSIONS}

Our results indicate that oscillating quintessence models, even for very simple potentials, can display more complex behavior than has previously been considered. Previous discussions have centered on the early oscillation case, in which the oscillation frequency is always much larger than the expansion rate when the scalar field dominates the expansion. Our results show that the late oscillation case, in which the scalar field transitions from a slowly rolling regime at early times into oscillations at late times, can also be consistent with current observations, although the behavior of these latter models 


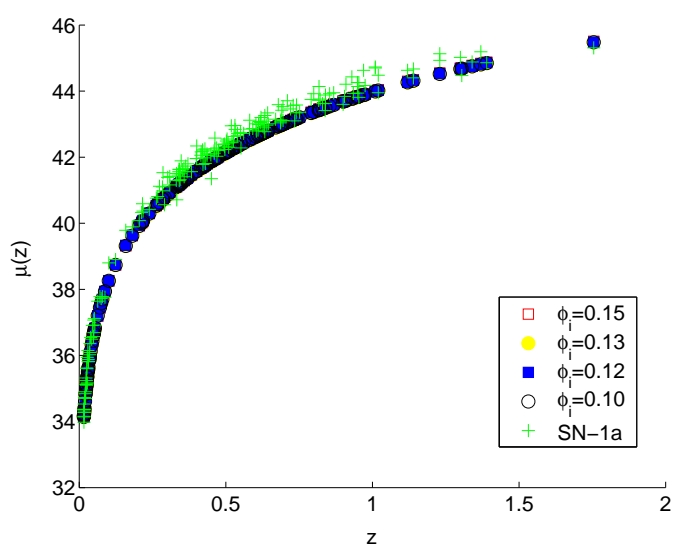

FIG. 5: Distance modulus for the indicated values of $\phi_{i}$ along with the supernova values used in our analysis, for $V(\phi)=$ $k|\phi|^{n}$, with $n=0.16$.

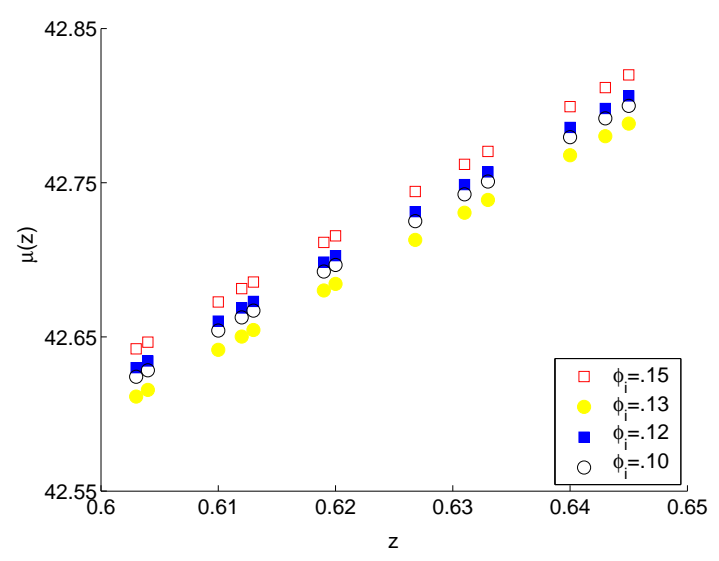

FIG. 6: A blow-up of Fig. 5] illustrating the difference in behavior of the four indicated models.

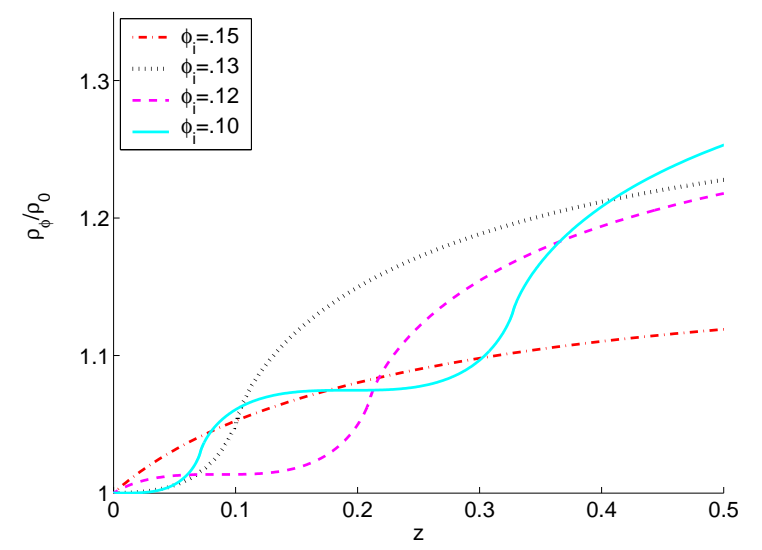

FIG. 7: Evolution of the scalar field density, normalized to unity at the present, as a function of redshift for the four models displayed in Figs. 2, 5, and 6 depends crucially on the oscillation phase of the scalar field at present. Johnson and Kamionkowksi [25] have argued recently that rapidly-oscillating scalar fields with a time-averaged value of $w_{\phi}$ less than 0 are unstable to the growth of inhomogeneities (see also the argument in Ref. [26]). In this case, only the late oscillation models would remain as acceptable oscillating scalar field models for dark energy.

How general are our results? The conclusion that $\nu / H$ increases with time depends only on the assumption that the scalar field energy density decays more slowly than the background energy density, so it should be generally applicable to any plausible model for dark energy. On the other hand, our comparison of models with observations was restricted to the case of power-law potentials. However, we would expect qualitatively similar results for other bound potentials with a single minimum at $V=0$. If the minimum of the potential were at $V_{0}>0$, the analysis is almost identical, except that the dark energy now has an additional component with constant density $V_{0}$. Such a model would have a values of $w(z)$ closer to -1 than the corresponding model with a minimum at $V=0$, and thus, would tend to agree more closely with current observations. One type of potential that could display completely different behavior from those studied here is a $V(\phi)$ with multiple minima, such as the Mexican hat potential discussed in Ref. [14]. In this case, the field evolution can be considerably more complex, as the field can initially oscillate over the entire range of the potential, but will eventually become trapped in a local minimum at late times. In this case the simple analysis presented here will not apply.

\section{Acknowledgments}

R.J.S. was supported in part by the Department of Energy (DE-FG05-85ER40226). 
[3] W.M. Wood-Vasey, et al., Astrophys. J. 666, 694 (2007).

[4] T.M. Davis, et al., Astrophys. J. 666, 716 (2007).

[5] E.J. Copeland, M. Sami, and S. Tsujikawa, Int. J. Mod. Phys. D 15, 1753 (2006).

[6] B. Ratra and P.J.E. Peebles, Phys. Rev. D 37, 3406 (1988).

[7] M.S. Turner and M. White, Phys. Rev. D 56, 4439 (1997).

[8] R.R. Caldwell, R. Dave, and P.J. Steinhardt, Phys. Rev. Lett. 80, 1582 (1998).

[9] A.R. Liddle and R.J. Scherrer, Phys. Rev. D 59, 023509 (1998).

[10] P.J. Steinhardt, L. Wang, and I. Zlatev, Phys. Rev. D 59, 123504 (1999).

[11] M.S. Turner, Phys. Rev. D 28, 1243 (1983).

[12] V. Sahni and L. Wang, Phys. Rev. D 62, 103517 (2000).

[13] S.D.H. Hsu, Phys. Lett. B 567, 9 (2003).

[14] E. Masso, F. Rota, and G. Zsembinszki, Phys. Rev. D 72, 084007 (2005).

[15] J.-A. Gu, arXiv:0711.3606.
[16] A. Kurek, O. Hrycyna, and M. Szydlowski, Phys. Lett. B 659, 14 (2008).

[17] T. Damour and V.F. Mukhanov, Phys. Rev. Lett. 80, 3440 (1998).

[18] S. Dodelson, M. Kaplinghat, E. Stewart, Phys. Rev. Lett. 85, 5276 (2000).

[19] G. Barenboim, O. Mena, and C. Quigg, Phys. Rev. D 71, 063533 (2005).

[20] G. Barenboim and J. Lykken, Phys. Lett. B 633, 453 (2006).

[21] B. Feng, M. Li, Y.-S. Piao, and X. Zhang, Phys. Lett. B 634, 101 (2006).

[22] E.V. Linder, Astropart. Phys. 25, 167 (2006).

[23] R.R. Caldwell and E.V. Linder, Phys. Rev. Lett. 95, 141301 (2005).

[24] R.J. Scherrer and A.A. Sen, Phys. Rev. D 77, 083515 (2008).

[25] M.C. Johnson and M. Kamionkowski, arXiv:0805.1748.

[26] S. Kasuya, Phys. Lett. B 515, 121 (2001). 